en by the presence of HIV or diabetes co-morbidities, which oriented the selection of 2 LAM M. tuberculosis isolates from TB patients with no comorbidities, but with different TB severities. Despite their close genetic structure, we are finding a distinctive pattern of cytokine production by human and mouse macrophages infected with either isolate. Interestingly, the high TB severity-associated isolate is a poor inducer of cytokine responses. Mechanistically, we relate this poor induction of cytokine production with a differential capacity of the bacteria in activating the host transcriptional machinery, as well as the inflammasome. Furthermore, a different in vivo progression of infection by the selected $M$. tuberculosis isolates was also observed. Most notably, the isolate associated with high TB severity showed higher dissemination patterns from the lung to the liver and spleen than that associated with mild TB. To investigate if the link TB severity-cytokine response was broader, we tested the cytokine response induced in human peripheral blood mononuclear cells by a series of other M. tuberculosis isolated from patients with different TB severities. A segregation of isolates was observed, with poor inducers of cytokine responses being generally associated with high TB severity. Collectively, our findings suggest that bacterial-intrinsic properties modulate the intensity of the initial immune response with likely consequences for the severity of TB. Our studies open interesting avenues for TB interventions, namely by raising the importance of considering the pathogen diversity when designing host-directed therapies.

6.44

doi: $10.15789 / 2220-7619-2018-4-6.44$

\section{THE ROLE OF THE IS6110 IN MICRO- AND MACROEVOLUTION OF MYCOBACTERIUM TUBERCULOSIS LINEAGE 2}

E. Shitikov' ${ }^{1}$ A. Guliaev ${ }^{1}$, J. Bespyatykh' ${ }^{1}$ I. Mokrousov ${ }^{2}$, E. Ilina ${ }^{1}$, V. Govorun ${ }^{1}$

${ }^{1}$ Federal Research and Clinical Centre of Physical-Chemical Medicine, Moscow, Russia $;{ }^{2}$ St. Petersburg Pasteur Institute,

St. Petersburg, Russia

Genomes of Mycobacterium tuberculosis complex members contain the insertion sequence (IS) 6110 which, due to its high quantitative and positional variability, has become a widely used marker in epidemiological studies. The element plays an important role in microorganism genome plasticity, but still many consequences and causes of transposition have not been fully described. This work studies the transposition mechanism of IS 6110 and its impact on the evolution of $M$. tuberculosis (Mtb).

Whole-genome sequencing data of 902 Mtb lineage 2 isolates was obtained from NCBI and ENA databases. Phylogenetic sublineages were determined based on SNP analysis (120 samples belonged to the ancient Beijing (17 proto-Beijing, 28 Asia Ancestral 1, 13 Asia Ancestral 2, 38 Asia Ancestral 3), 782 samples belonged to the modern Beijing (10 Asian African 1, 29 Asian African 3, 65 Asian African 2, 43 Pacific RD150, 140 Europe/Russia W148 outbreak, 361 Central Asia) (E. Shitikov et al., SciRep, 2017). ISMapper was used to determine the sites of integration of the IS6110 (Hawkey et al., BMC Genomics, 2015).

We obtained 17972 points of insertion, which belonged to 865 independent positions in the H37Rv genome. The mean copy number per genome was 19.92 (from 9 to 25). To describe the evolution of an element in the genome, we arranged our samples in the order corresponding to a phylogenetic tree constructed on the basis of SNPs. We determined the stepwise mechanism of transposition, in which the transition to a new subpopulation is accompanied by a change in the localization of several copies of IS. It is important to note that the localization of the element in the ancestral population does not change, which implies a transposition only by "copypaste" mechanism. In addition, we defined genes (537 sites (256 genes)) and intergenic regions (328 sites), where the element was integrated. Sixteen genes previously identified as being essential under different experimental conditions were found to contain IS. Further we carried out identification of IS6110 mediated LSPs which showed the presence of recombination events (deletion) between inversely oriented elements.

In conclusion, we determined the evolution and role of IS 6110 for Mtb lineage 2 strains. We identified evolutionary and subpopulation-specific sites of integration which can be used for typing and subsequent research.

The work was supported by the grant from the Russian Foundation for Basic Research RFBR 18-04-01035.

NGS DETERMINATION OF MYCOBACTERIAL TRANSRENAL DNA AS POTENTIAL TOOL OF CLINICAL DIAGNOSTIC

V. Sinkov ${ }^{1}$, O. Ogarkov ${ }^{1,2}$, A. Plotnikov ${ }^{3}$, S. Zhdanova ${ }^{1}$, N. Belkova ${ }^{4}$, M. Koshcheev ${ }^{5}$, S. Heysell ${ }^{6}$

${ }^{1}$ SC FHHRP, Irkutsk, Russia; ${ }^{2}$ ISMACE, Irkutsk, Russia; ${ }^{3}$ ICIS UD RAS, Orenburg, Russia: ${ }^{4}$ LIN SB RAN

Irkutsk, Russia; ${ }^{5}$ RTBH, Irkutsk, Russia; ${ }^{6} U V A$, Charlottesville, $V A, U S A$

Sputum is a major object for monitoring TB treatment and diagnostics it is strictly depended from bacterial load. There is a substantial need for less variable and more reliable specimen for the diagnosis of tuberculosis and for treatment monitoring. The objective of this study is to estimation diagnostic power of full genome sequencing (NGS) of soluble mycobacterial transrenal DNA (mtr-DNA) in urine of TB patients and TaqMan tests designed after analysis of metagenomic data.

DNA patient with pulmonary tuberculosis (TB) isolated from $4 \mathrm{ml}$. of urine by QIAamp Circulating Nucleic Acid Kit. Detection of TB positive samples made by previously developed PCR targeted to 45 base pairs fragment of mycobacterial genome. It was chosen 2 positive PCR samples. It were mapped on the reference genome $M$. $t u$ berculosis (NC 000962.3) by BWA. In total were mapped 16579 paired reads of the one sample $(0.83 \%)$ and 1783754 (64\%) of the second sample respectively. There were also analyzed mapped DNA sequences with more than $4 \mathrm{x}$ coverage. The median length of mtr-DNA found as $20 \mathrm{bp}$.

It was found $156 \mathrm{mtr}$-DNA fragments repeated in both samples. The median length of DNA fragment was found was $20 \mathrm{bp}$. Five fragments including part of $16 \mathrm{~S}$ rRNA gene were chosen for design primer and TaqMan probes for targets from 43 to $60 \mathrm{bp}$. Length of primers and probes were reduced by Locked Nucleic Acid (LNA) bases. The sensitivity and specificity of the developed tests was determined by known DNA samples from urine. The result obtained did not reveal a significant improvement in the sensitivity of the new tests. PCR-RT cutoff remained approximately 40 cycles, like in previously developed tests.

High specificity and sensitivity of NGS and low of PCR suggest that diagnosis and monitoring of tuberculosis by mtr-DNA should be based on NGS, rather than on PCR.

We acknowledge support from the RFBR-NIH grant \#1754-30020. 